\title{
Study of hTERT and Histone 3 Mutations in Medulloblastoma
}

\author{
Marta Viana-Pereira ${ }^{a}$ b Gisele Caravina Almeida ${ }^{d}$ \\ João Norberto Stavale ${ }^{g}$ Susana Malheiro $^{\text {h }}$ Carlos Clara $^{\text {e }}$ Patrícia Lobo $^{c}$ \\ José Pimentel ${ }^{c}$ Rui Manuel Reis ${ }^{a}, b, f$ \\ a Life and Health Sciences Research Institute (ICVS), School of Health Sciences, University of Minho, Braga, \\ bICVS/3B's - PT Government Associate Laboratory, Braga/Guimarães, and 'Laboratory of Neuropathology, \\ Santa Maria Hospital, Institute of Molecular Medicine, Lisbon Faculty of Medicine, Lisbon, Portugal; Departments of \\ dPathology and ${ }^{\mathrm{d}}$ Neurosurgery and ${ }^{\mathrm{f}}$ Molecular Oncology Research Center, Barretos Cancer Hospital, Barretos, and \\ Departments of ${ }^{9}$ Pathology and ${ }^{\mathrm{h}}$ Neurology and Neurosurgery, Federal University of São Paulo, São Paulo, Brazil
}

\section{Key Words}

hTERT $\cdot$ Mutations $\cdot$ Medulloblastoma $\cdot$ Biomarkers

\begin{abstract}
Hotspot activating mutations of the telomerase reverse transcriptase (hTERT) promoter region were recently described in several tumor types. These mutations lead to enhanced expression of telomerase, being responsible for telomere maintenance and allowing continuous cell division. Additionally, there are alternative telomere maintenance mechanisms, associated with histone $\mathrm{H} 3$ mutations, responsible for disrupting the histone code and affecting the regulation of transcription. Here, we investigated the clinical relevance of these mechanistically related molecules in medulloblastoma. Sixty-nine medulloblastomas, formalin fixed and paraffin embedded, from a cohort of patients aged 1.5-70 years, were used to investigate the hotspot mutations of the $h T E R T$ promoter region, i.e. H3F3A and HIST1H3B, using Sanger sequencing. We successfully sequenced $h T E R T$ in all 69 medulloblastoma samples and identified a total of 19 mutated cases (27.5\%). c.-124:G>A and c.-146:G>A mutations were detected, respectively, in 16 and 3 samples. Similar to previous
\end{abstract}

reports, hTERT mutations were more frequent in older patients $(p<0.0001)$, being found only in 5 patients $<20$ years of age. In addition, $h T E R T$-mutated tumors were more frequently recurrent $(p=0.026)$ and $h T E R T$ mutations were significantly enriched in tumors located in the right cerebellar hemisphere $(p=0.039)$. No mutations were found on the H3F3A or HIST1H3B genes. hTERT promoter mutations are frequent in medulloblastoma and are associated with older patients, prone to recurrence and located in the right cerebellar hemisphere. On the other hand, histone 3 mutations do not seem to be present in medulloblastoma.

๑) 2016 S. Karger AG, Basel

\section{Introduction}

Shortening of telomeres is a natural mechanism that occurs during the replication of somatic cells, and is responsible for cell aging and replicative senescence $[1,2]$. One major mechanism of telomere length control is associated with the activity of the telomerase enzyme $[1,2]$. The catalytic subunit of this enzyme is encoded by the telomerase reverse transcriptase (hTERT) gene and mod-

\section{KARGER}

(c) 2016 S. Karger AG, Basel

E-Mail karger@karger.com

www.karger.com/pat
Rui Manuel Reis, $\mathrm{PhD}$

Life and Health Sciences Research Institute (ICVS), School of Health Sciences University of Minho, Campus de Gualtar

PT-4710-057 Braga (Portugal)

E-Mail rreis @ecsaude.uminho.pt 
ulates telomerase activity $[2,3]$. Although telomerase is normally silenced in somatic cells, it is active in male germ cells, activated lymphocytes, stem cell populations and during development [4-6]. In the large majority of human cancers, abnormal telomerase reactivation is observed, leading to telomere maintenance and limitless proliferative potential [7-9].

Hotspot activating mutations were recently described in the $h T E R T$ promoter region in a variety tumor types, including medulloblastomas (20\%) and glioblastomas (83\%) among many others [10-12]. These mutations are located in 2 positions, c.-124:G>A and c.-146:G>A, and are thought to be the main biological mechanism responsible for telomerase overexpression $[13,14]$. The functional consequence of the presence of promoter mutations was initially associated with the creation a new binding motif for E-twenty-six (ETS) transcription factors and ternary complex factors (TCFs) near the transcription start, leading to an increased transcription of the $h T E R T$ promoter of at least 2 -fold, as assessed by reporter assays $[13,14]$. Recently, it was also demonstrated that the occurrence of these mutations leads to the recruitment of the GABP transcription factor, and the activation of transcription and aberrant expression of $h T E R T$ in several cancers [15]. In addition to these mutations, a common functional single nucleotide polymorphism (SNP), rs2853669, located in the hTERT promoter has been shown to influence the prognostic value of the mutations mentioned in gliomas and bladder and renal cell carcinomas [16].

Alternative lengthening of telomeres (ALT) pathways has also been described, being present in 15\% of cancers. Inactivating $A T R X$ mutations, associated with histone $\mathrm{H} 3$ mutations, were described in tumors with ALT phenotype, including $1.5 \%$ of medulloblastoma [17]. These mutations were reported to be responsible for disrupting the histone code and affecting the regulation of transcription [18]. Mutations in histone 3 (H3F3A, encoding H3.3 and HIST1H3B, encoding H3.1) have been reported in higher frequencies in pediatric gliomas of different histologies, but have not been extensively studied in medulloblastoma [18-20].

In this work, we investigated the clinical relevance of these important regulators in medulloblastoma, confirming that $h T E R T$ promoter mutations are a frequent event in medulloblastoma. These mutations were associated with older age, tumor recurrence and tumors from the right cerebellar hemisphere. On the other hand, histone mutations do not seem to be present in this type of brain tumor.

hTERT and Histone 3 Mutations in Medulloblastoma

\section{Material and Methods}

\section{Tumor Samples and DNA Isolation}

Formalin-fixed, paraffin-embedded (FFPE) medulloblastoma samples from 68 patients, i.e. 68 primary tumors and 1 recurrence (with a matched primary tumor sample) aged 1.5-70 years, were obtained after approval by local and multicenter ethical review committees at the Laboratory of Neuropathology, Hospital of Santa Maria (CHLN), Lisbon, Portugal, and the Federal University of São Paulo, São Paulo, and Barretos Cancer Hospital, Barretos, Brazil. All the samples enrolled in the study were unlinked and unidentified from their donors. Due the retrospective nature of the study, no written informed consent from patients was obtained. The presence of tumor tissue in the samples and the tumor histology was verified on an $\mathrm{H} \& \mathrm{E}$-stained section by the pathologists J.N.S., J.P. and G.C.A.

DNA was isolated using the QIAamp DNA mini kit (Qiagen) according to the manufacturer's instructions and as previously reported [21].

\section{Mutation Analysis}

$h T E R T$ promoter mutations were screened using the primers previously described [22] that amplify the sites of the c.-124:G>A and c.-146:G>A mutations, as previously described [12, 23, 24].

Histone 3 hotspot regions previously identified $[18,19]$ were amplified with the following primers: $H 3 F 3 A$ (forward: $5^{\prime}$-CATGGCTCGTACAAAGCAGA; reverse: $5^{\prime}$-CAAGAGAGACTTTGTCCCATTTTT) and HIST1H3B (forward: 5'-TTTCCTTTCCTCCACAGACG; reverse: $5^{\prime}$-CGGTAACGGTGAGGCTTTT).

Amplification of PCR products was confirmed by gel electrophoresis. Subsequently, samples were Sanger-sequenced after PCR product purification with ExoStar (Invitrogen). Sequencing PCR was performed using the ABI PRISM 3730xL DNA analyzer (Applied Biosystems, USA) by STAB VIDA services (Portugal).

\section{Statistical Analysis}

All statistical tests were done in SPSS v20.0 (SPSS Inc.). Correlations between categorical values were done using the 2-tailed $\chi^{2}$ test and the Fisher exact test. Continuous variables were assessed using the Mann-Whitney U test. Associations between hTERT mutational status and patients' overall survival were analyzed using the Kaplan-Meier method and the log-rank test. A p value of $<0.05$ was considered significant.

\section{Results}

\section{hTERT Is Frequently Mutated in Medulloblastoma}

We successfully sequenced $h T E R T$ in 69 FFPE medulloblastomas, corresponding to 68 primary tumors and 1 recurrence. Of these, 35 samples were from pediatric patients (1.5-17 years of age) and 32 from adults ( $>18$ years); for an additional sample we could not confirm the age at diagnosis. We identified a total of 19 cases $(27.5 \%)$ with hTERT promoter mutations (table 1). The c.-124:G>A mutation was detected in 16 samples, and the c.-146:G>A mutation (fig. 1) in 3 cases.

Pathobiology 2017;84:108-113 
Table 1. Clinicopathological and molecular features of medulloblastoma

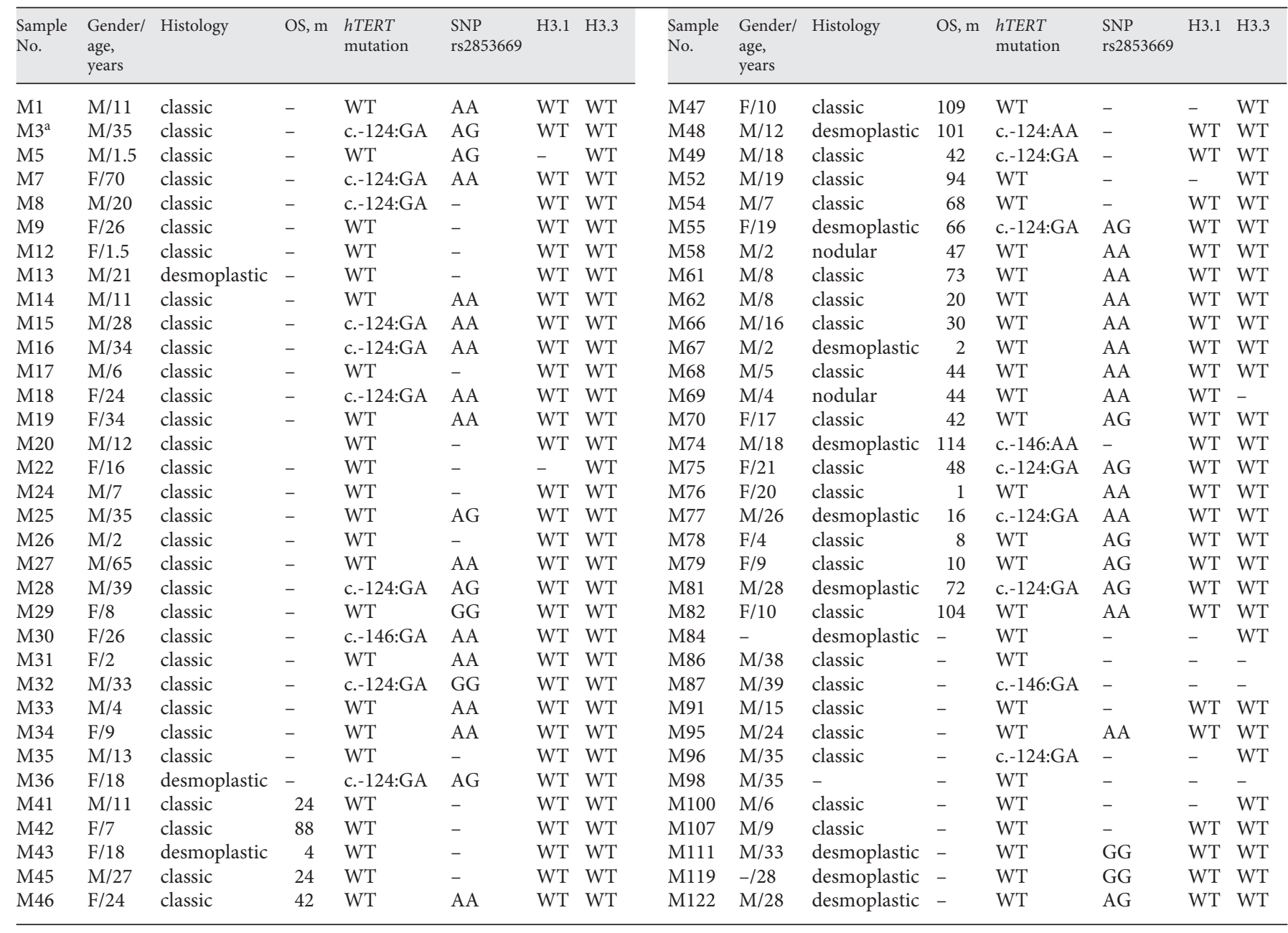

Genotypes for $h$ TERT mutations: WT for both c.-124:G>A and c.-146:G>A mutations, c.-124:GA (heterozygous mutant), c.-124:AA (homozygous mutant), c.-146:GA (heterozygous mutant) and c.-146:AA (homozygous mutant). Genotypes for $h T E R T$ rs2853669 SNP: AA (WT), AG (heterozygous mutant) and GG (homozygous mutant). $-=$ - Data not available; $\mathrm{m}=$ months; $\mathrm{OS}=$ overall survival.

a The same mutational status was seen in the primary and the recurrent sample of this case.

Similarly to previous reports $[10,11], h T E R T$ mutations were more frequent in older patients (table $2 ; \mathrm{p}<$ 0.0001 ), being found in only 3 patients younger than 17 years $(3 / 35,8.6 \%)$, whereas $>50 \%$ of the adult patients presented with $h T E R T$ promoter mutations (18/32, $56.3 \%)$. In addition, $h T E R T$-mutated tumors were more frequently in the recurrent cases $(\mathrm{p}=0.026)$, and $h T E R T$ mutations were significantly enriched in the tumors located in the right cerebellar hemisphere $(\mathrm{p}=0.039)$. No other clinicopathological features were found to be statistically significant (table $2 ; \mathrm{p}>0.05$ ).

The polymorphism rs2853669 was successfully sequenced in 41 of the 69 samples, and was present in $41.5 \%$ of the cases. Although this polymorphism has been described as modifying the survival of mutant carriers [16], we could not confirm these results in our cohort as only 6 samples presenting the variant also had survival data. No differences were seen between wild-type (WT) and mutated samples regarding overall survival (fig. $2 ; \mathrm{p}=0.200$ ).

\section{Histone Mutations Are Rare Events in Medulloblastoma}

We designed primers for the hotspot regions of $\mathrm{H} 3 \mathrm{~F} 3 \mathrm{~A}$ and $H I S T 1 H 3 B$ and successfully sequenced 59 samples for HIST $1 H 3 B$ and 65 for $H 3 F 3 A$. None of the samples analyzed harbored any mutation on $\mathrm{H} 3 \mathrm{~F} 3 \mathrm{~A}$ or $\mathrm{HIST} 1 \mathrm{H} 3 \mathrm{~B}$. 


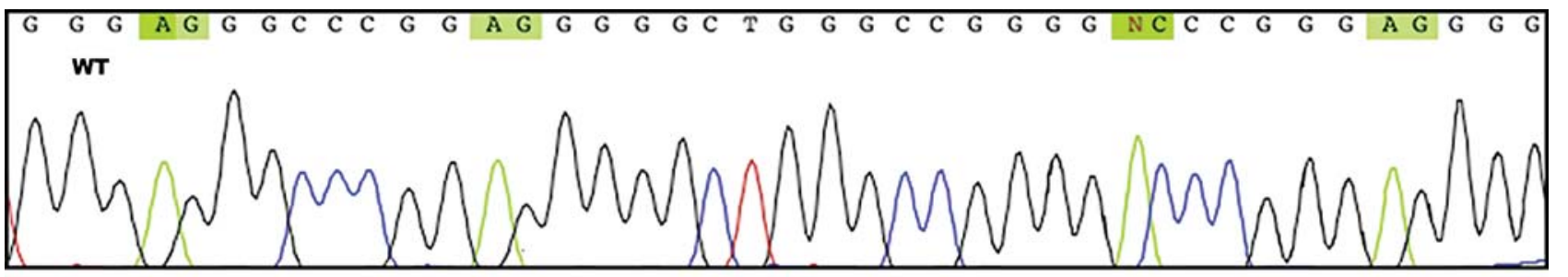

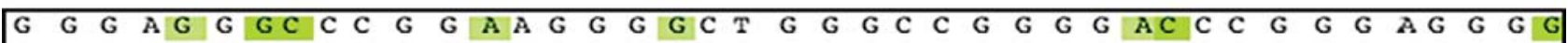
C.-124:G>A
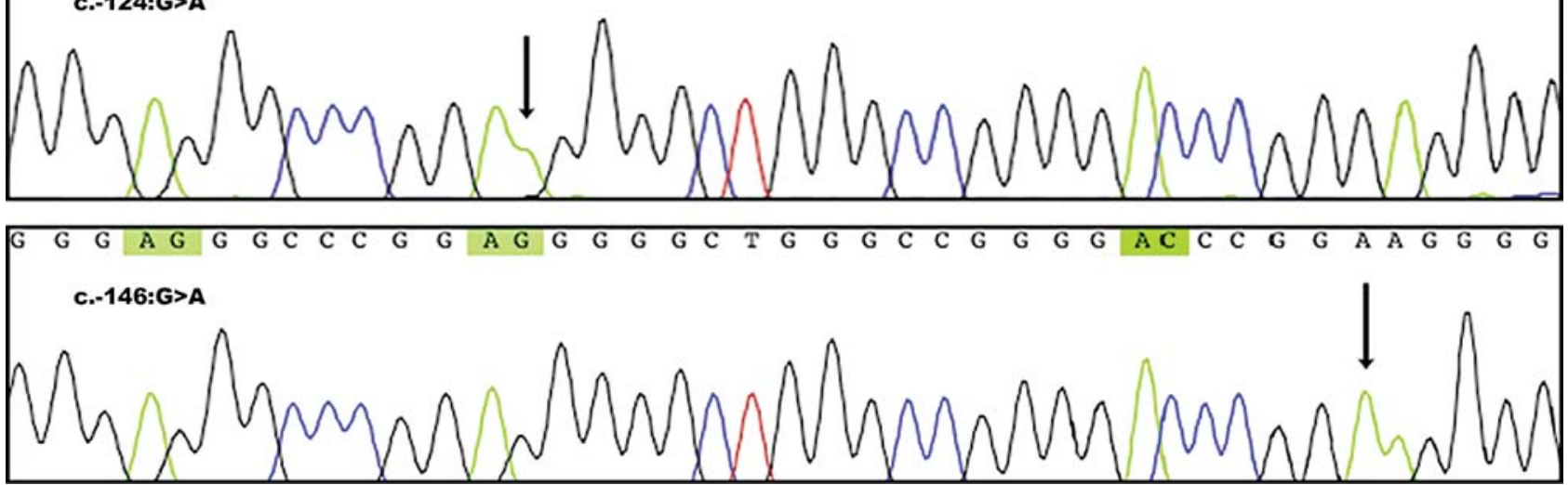

Fig. 1. Representative electropherogram traces of $h$ TERT Sanger sequencing from a WT sample and c.-124:G>A and c.-146:G>A mutant cases. Arrows indicate the mutated nucleotide.

\section{Discussion}

Promoter mutations of the $h T E R T$ gene were first described in melanoma in 2 distinct reports $[13,14]$. Since then, $h T E R T$ promoter mutations have been described across several tumor types, and are one of the most frequent genetic alterations involved in human tumorigenesis $[12,25]$.

In this study, we demonstrate that $28 \%$ of our medulloblastoma samples presented $h T E R T$ hotspot promoter mutations, in line with previous reports $(18-33 \%)[10,11$, $25,26]$. These mutations were more frequent in older patients $(\mathrm{p}<0.0001)$, were not seen in any infants (aged $<4$ years) and were only present in 3 children of 12,14 and 16 years of age. Previous reports have shown the same trend, with Koelsche et al. [26] reporting 3 and $65 \%$ of these mutations, respectively, in pediatric and adult patients. This is line with the literature that demonstrates clinical and molecular characteristics distinguishing pediatric and adult medulloblastomas [27]. hTERT mutations have been described to be associated with less metastatic medulloblastoma at diagnosis [10], and we have shown that these tumors were more prone to recurrence

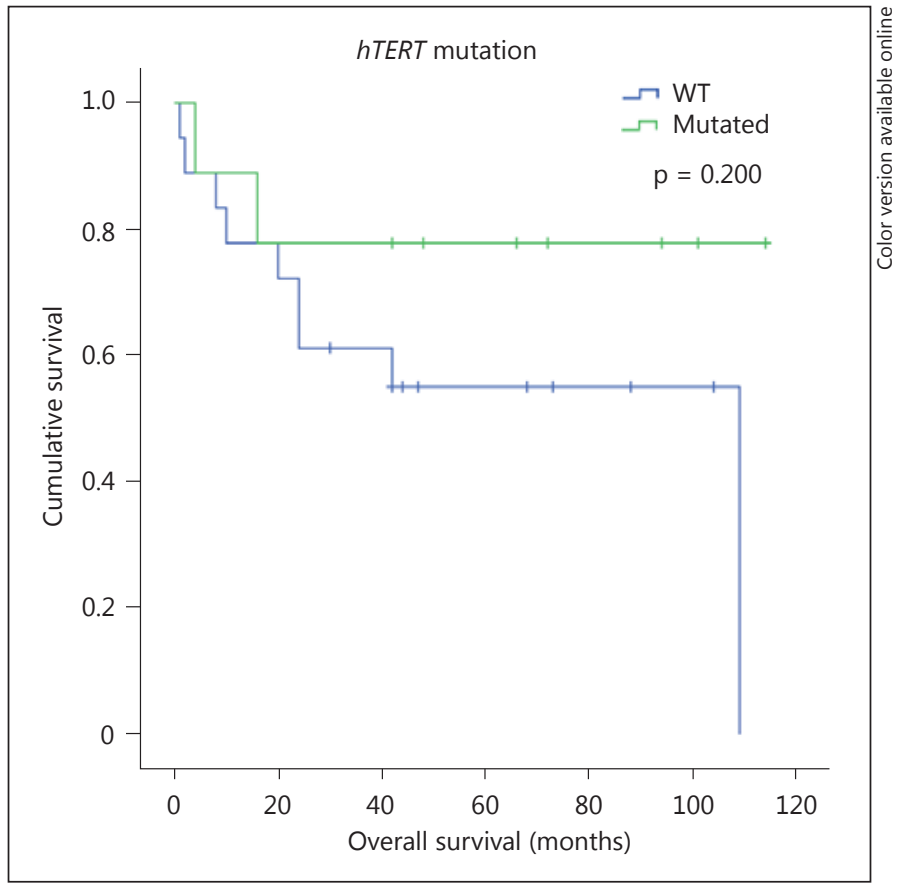

Fig. 2. Kaplan-Meier survival curves for hTERT WT and mutant samples. No statistical differences were seen between the 2 groups ( $\mathrm{p}=0.200$, log-rank test). 
Table 2. Association of $h T E R T$ mutation with clinicopathological features of medulloblastoma

\begin{tabular}{|c|c|c|c|}
\hline Characteristic & $\begin{array}{l}\text { hTERT } \\
\text { WT }\end{array}$ & $\begin{array}{l}\text { hTERT } \\
\text { mutation }\end{array}$ & $\mathrm{p}$ value \\
\hline \multicolumn{4}{|l|}{ Age, years } \\
\hline Median & 11 & 26 & \multirow[t]{3}{*}{$<0.0001^{\mathrm{a}}$} \\
\hline Range & $1.5-65$ & $12-70$ & \\
\hline n.a. & 1 & 1 & \\
\hline \multicolumn{4}{|l|}{ Gender } \\
\hline Female & 15 & 7 & \multirow[t]{3}{*}{$1.000^{c}$} \\
\hline Male & 30 & 14 & \\
\hline n.a. & 2 & 1 & \\
\hline \multicolumn{4}{|l|}{ Histology } \\
\hline Classic & 38 & 14 & \multirow[t]{5}{*}{$0.108^{c}$} \\
\hline Desmoplastic & 6 & 7 & \\
\hline Anaplastic & 0 & 0 & \\
\hline Nodular & 2 & 0 & \\
\hline n.a. & 1 & 1 & \\
\hline \multicolumn{4}{|l|}{ Metastasis } \\
\hline Negative & 16 & 9 & \multirow[t]{3}{*}{$0.286^{\mathrm{b}}$} \\
\hline Positive & 5 & 0 & \\
\hline n.a. & 26 & 13 & \\
\hline \multicolumn{4}{|l|}{ Recurrence } \\
\hline Negative & 18 & 8 & \multirow[t]{3}{*}{$0.026^{c}$} \\
\hline Positive & 2 & 6 & \\
\hline n.a. & 27 & 8 & \\
\hline \multicolumn{4}{|l|}{ Location } \\
\hline $\begin{array}{c}\text { Cerebellar vermis }+ \\
\text { 4th ventricle }\end{array}$ & 26 & 8 & \multirow[t]{4}{*}{$0.039^{\mathrm{b}}$} \\
\hline Right cerebellar hemisphere & 4 & 7 & \\
\hline Left cerebellar hemisphere & 4 & 3 & \\
\hline n.a. & 13 & 4 & \\
\hline
\end{tabular}

( $\mathrm{p}=0.026)$ compared to the hTERT WT medulloblastomas. In addition, we also demonstrate, for the first time, that hTERT mutations were significantly enriched in tumors arising in the right cerebellar hemisphere ( $\mathrm{p}=$ 0.039 ).
Mutations in histone 3 leading to K27M and G34R/ G34V have been extensively reported in pediatric highgrade gliomas $[18,19]$. In spite of this, not many works have reported the results of these mutations in medulloblastomas. In 2014, Huether et al. [20] studied $>1,000$ different pediatric cancers, 70 of which were medulloblastomas, and did not see histone mutations in this brain tumor type. Similarly, we successfully sequenced approximately 60 samples for the hotspot mutations of HIST $1 H 3 B$ and H3F3A and found no mutations in our samples. Currently, it is well accepted that medulloblastomas can be cytogenetically and molecularly stratified into distinct subgroups that are associated with distinct clinicopathological features $[28,29]$. hTERT mutations have been reported more frequently in the $\mathrm{SHH}$ molecular subgroup [10], but further studies are needed to validate whether they are associated with particular cytogenetic and molecular subtypes.

In conclusion, we reported that hotspot $h T E R T$ mutations are present in approximately $1 / 3$ of medulloblastomas cases and are associated with older patients and recurrent tumors, constituting a potential biomarker for medulloblastomas.

\section{Acknowledgements}

This study was partially supported by $\mathrm{CNPq} /$ Universal (475358/2011-2), Fundação de Amparo à Pesquisa do Estado de São Paulo (FAPESP; 2012/19590-0) and Fundação para a Ciência e Tecnologia (FCT; PTDC/SAU-ONC/115513/2009) grants to R.M.R. The project was cofinanced by Programa Operacional Regional do Norte (ON.2-O Novo Norte), Quadro de Referência Estratégico Nacional (QREN) and Fundo Europeu de Desenvolvimento Regional (FEDER). M.V.-P. is the recipient of an FCT Post-Doctorate Research Fellowship (SFRH/BPD/104290/2014).

\section{Disclosure Statement}

The authors disclose no potential conflicts of interest.

\section{References}

1 Bryan TM, Cech TR: Telomerase and the maintenance of chromosome ends. Curr Opin Cell Biol 1999;11:318-324.

2 Cong YS, Wright WE, Shay JW: Human telomerase and its regulation. Microbiol Mol Biol Rev 2002;66:407-425.

3 Weinrich SL, Pruzan R, Ma L, et al: Reconstitution of human telomerase with the template RNA component hTR and the catalytic protein subunit hTRT. Nat Genet 1997;17:498502.
4 Kim NW, Piatyszek MA, Prowse KR, Harley $\mathrm{CB}$, West MD, Ho PL, Coviello GM, Wright WE, Weinrich SL, Shay JW: Specific association of human telomerase activity with immortal cells and cancer. Science 1994;266: 2011-2015.

5 Wright WE, Piatyszek MA, Rainey WE, Byrd W, Shay JW: Telomerase activity in human germline and embryonic tissues and cells. Dev Genet 1996;18:173-179. 
6 Ozturk S, Sozen B, Demir N: Telomere length and telomerase activity during oocyte maturation and early embryo development in mammalian species. Mol Hum Reprod 2014; 20:15-30.

7 Blasco MA, Hahn WC: Evolving views of telomerase and cancer. Trends Cell Biol 2003; 13:289-294.

8 Ding D, Zhou J, Wang M, Cong YS: Implications of telomere-independent activities of telomerase reverse transcriptase in human cancer. FEBS J 2013;280:3205-3211.

9 Shay JW, Bacchetti S: A survey of telomerase activity in human cancer. Eur J Cancer 1997; 33:787-791.

10 Remke M, Ramaswamy V, Peacock J, et al: TERT promoter mutations are highly recurrent in SHH subgroup medulloblastoma. Acta Neuropathol 2013;126:917-929.

11 Killela PJ, Reitman ZJ, Jiao Y, et al: TERT promoter mutations occur frequently in gliomas and a subset of tumors derived from cells with low rates of self-renewal. Proc Natl Acad Sci USA 2013;110:6021-6026.

12 Vinagre J, Almeida A, Pópulo H, et al: Frequency of TERT promoter mutations in human cancers. Nat Commun 2013;4:2185.

13 Horn S, Figl A, Rachakonda PS, et al: TERT promoter mutations in familial and sporadic melanoma. Science 2013;339:959-961.

14 Huang FW, Hodis E, Xu MJ, Kryukov GV, Chin L, Garraway LA: Highly recurrent TERT promoter mutations in human melanoma. Science 2013;339:957-959.
15 Bell RJ, Rube HT, Kreig A, et al: The transcription factor GABP selectively binds and activates the mutant TERT promoter in cancer. Science 2015;348:1036-1039.

16 Rachakonda PS, Hosen I, de Verdier PJ, et al: TERT promoter mutations in bladder cancer affect patient survival and disease recurrence through modification by a common polymorphism. Proc Natl Acad Sci USA 2013;110: 17426-17431.

17 Heaphy CM, de Wilde RF, Jiao Y, et al: Altered telomeres in tumors with $A T R X$ and DAXX mutations. Science 2011;333:425.

18 Schwartzentruber J, Korshunov A, Liu XY, et al: Driver mutations in histone H3.3 and chromatin remodelling genes in paediatric glioblastoma. Nature 2012;482:226-231.

19 Wu G, Broniscer A, McEachron TA, et al: Somatic histone $\mathrm{H} 3$ alterations in pediatric diffuse intrinsic pontine gliomas and non-brainstem glioblastomas. Nat Genet 2012;44:251253.

20 Huether R, Dong L, Chen X, et al: The landscape of somatic mutations in epigenetic regulators across 1,000 paediatric cancer genomes. Nat Commun 2014;5:3630.

21 Martinho O, Gouveia A, Viana-Pereira M, Silva P, Pimenta A, Reis RM, Lopes JM: Low frequency of MAP kinase pathway alterations in KIT and PDGFRA wild-type GISTs. Histopathology 2009;55:53-62.

22 Liu X, Wu G, Shan Y, Hartmann C, von Deimling A, Xing M: Highly prevalent TERT promoter mutations in bladder cancer and glioblastoma. Cell Cycle 2013;12:1637-1638.
23 Campanella NC, Celestino R, Pestana A, et al: Low frequency of TERT promoter mutations in gastrointestinal stromal tumors (GISTs). Eur J Hum Genet 2015;23:877-879.

24 Batista R, Cruvinel-Carloni A, Vinagre J, et al: The prognostic impact of TERT promoter mutations in glioblastomas is modified by the rs2853669 single nucleotide polymorphism. Int J Cancer 2016;139:414-423.

25 Huang DS, Wang Z, He XJ, et al: Recurrent TERT promoter mutations identified in a large-scale study of multiple tumour types are associated with increased TERT expression and telomerase activation. Eur J Cancer 2015; 51:969-976.

26 Koelsche C, Sahm F, Capper D, et al: Distribution of TERT promoter mutations in pediatric and adult tumors of the nervous system. Acta Neuropathol 2013;126:907-915.

27 Riffaud L, Hénaux PL: Prognostic factors of adult medulloblastomas: a review of the literature and perspectives. Neurochirurgie 2016;62:46-52.

28 Shih DJ, Northcott PA, Remke M, et al: Cytogenetic prognostication within medulloblastoma subgroups. J Clin Oncol 2014;32:886896.

29 Northcott PA, Shih DJ, Remke M, et al: Rapid, reliable, and reproducible molecular subgrouping of clinical medulloblastoma samples. Acta Neuropathol 2012;123:615-626. 